\title{
A case of foreign body granuloma masquerading as a soft tissue tumour
}

\author{
Henry Tan Chor Lip ${ }^{1,2,3}$, Tan Jih Huei ${ }^{1,2,3}$, Tuan Nur' Azmah Tuan Mat ${ }^{3}$ \\ 1 Pusat Perubatan Universiti Kebangsaan Malaysia. \\ 2 Clinical Research Centre, Hospital Sultan Ismail, Malaysia. \\ 3 General Surgery Department, Hospital Sultanah Aminah,Malaysia.
}

Keywords: Granuloma; foreign body; hernia; inguinal

\section{Introduction}

A foreign body granuloma is defined as a mass that forms at site of surgery due to biological tissue reaction to foreign material in the tissue [1]. It is a rare complication of inguinal hernioplasty and its incidence is still unknown due to lack of reports on such cases. The presentation may vary from simple superficial skin infection (SSI) to a fungating mass mimicking soft tissue malignancy. This report describes a man that presented with a fungating mass over the left inguinal region one year after inguinal hernioplasty.

\section{Case presentation}

A 42 year old male had bilateral inguinal hernioplasty with mesh repair performed in June 2015. The surgery was uneventful and had no post-operative surgical site infection (SSI). He presented again to our surgical clinic one year later for a fungating mass over the left inguinal region. Clinical examination revealed a fungating mass over the site of incision of previous hernioplasty scar (Figure 1A). The mass measured $3 \times 3 \mathrm{~cm}$, was hard in consistency, fixed to underlying tissue and had raw areas mixed with necrotic slough. He denied any infective symptoms of fever, skin redness or pus discharge prior to this. The full blood count was within normal range without leucocytosis. These findings made us suspect a soft tissue tumour (ie liposarcoma or desmoid tumour) or a squamous cell carcinoma of the skin.

An urgent computed tomography (CT) scan of abdomen showed a heterogenous, fungating soft tissue swelling within the left inguinal region. The mass involved the subcutaneous layer, external oblique aponeurosis, rectus abdominis muscle and was abutting the lateral side of urinary bladder (Figure 1B). There were also streaky densities in the fat surrounding the mass with increased neovascularization. The mass was seen to abut the patent femoral vessels laterally. From the CT report, the possible differential diagnosis were of soft tissue

Correspondence: Henry Tan Chor Lip

E-mail: relos1402@gmail.com

Received: 09-07-2017 Accepted: 30-08-2017

(iD) http://orcid.org/0000-0003-0621-9276

DOI: http://doi.org/10.4038/sljs.v35i3.8410
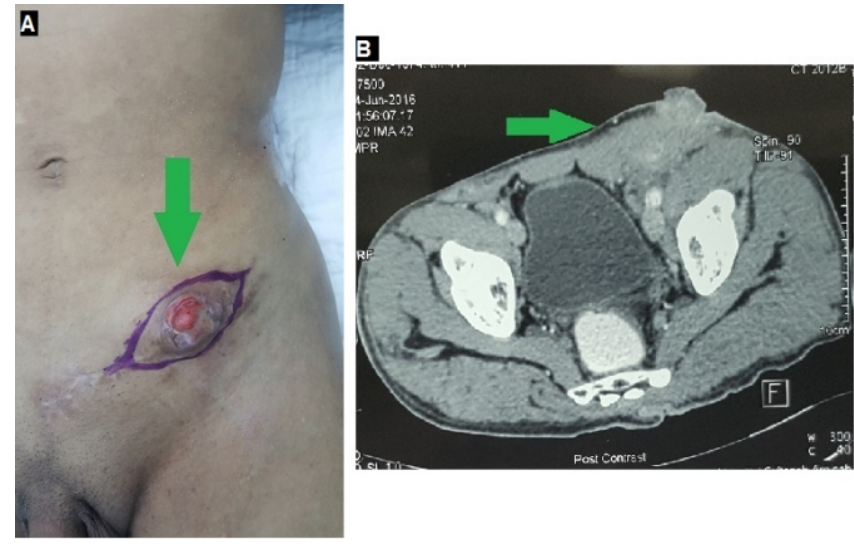

Figure 1: A. Pre-operative marking of the fungating mass over the left inguinal region (green arrow). B. CT abdomen with fungating mass (green arrow) infiltrating the surrounding subcutaneous and abdominal wall muscle.

tumour or skin neoplasm.

Histology of the wedge biopsy revealed pseudoepitheliomatous hyperplasia, underlying dermal fibrosis with infiltration of foamy histiocytes, lymphocytes and plasma cells. There were no granuloma or malignant cells. These features were consistent with a chronic inflammatory histology. Based on the suspicious findings on the CT scan, the patient underwent a wide local excision. The tumour was excised with clear margins.

At the base of the mass, a part of the mesh which had shrunk was identified and excised (Figure 2A, B, C ). It was evident intra-operatively that the chronic inflammatory reactions to the mesh led to the formation of the foreign body granuloma (Figure 2D). Post-operative period was uneventful. Patient was well at the review 3 weeks after surgery.

The gross morphology of the resected specimen measured $4.2 \times 2.8 \times 1.3 \mathrm{~cm}$ with a raised polypoidal skin lesion measuring $3.5 \times 2.5 \times 0.5 \mathrm{~cm}$. Cut section revealed a grey coloured surface and was solid in consistency. On microscopy, the sections exhibited focal granulation tissue formation with moderate foamy macrophages and neutrophils. These features were consistent with an infected suture granuloma without any presence of malignancy. 


\section{Discussion}

The Lictenstein inguinal hernia repair technique has been practiced for more than 50 year. Approximately one million meshes are used in inguinal hernia repair annually [2]. Nagar et al reported an incidence of suture granuloma of $0.3 \%$ from a retrospective study of 2447 paediatric herniotomy [3]. Incidences of paravesical granuloma after inguinal hernioplasty can be found dated back to 1959 by Brand et al. Subsequently 3 more similar cases were reported by Kise et al in $1999[1,4]$.

Foreign body granuloma may occur 0.5-11 years after inguinal hernioplasty. Surgical site infection was seen in the majority of cases of foreign body granuloma. This prolonged infectivity with pus discharge forms a chronic wound. Chronic inflammation due to mesh placement also predisposes to squamous cell carcinoma as reported by Birolini et al. Diagnosis of a malignancy secondary to chronic inflammatory changes had been straightforward in both reported cases with a history of chronic inflammatory wounds

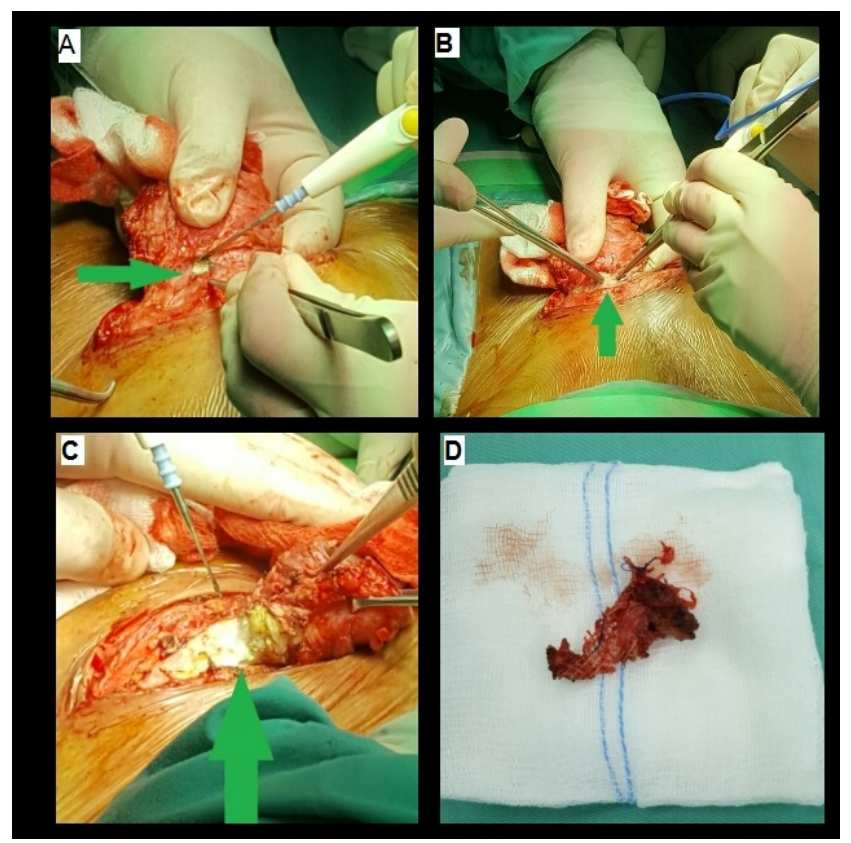

Figure 2: A. Dissection of the base of the mass exposing the underlying mesh (green arrow).

$B, C$. Dissection of the mass at the base which includes fibrosed tissues surrounding the mesh.

D. Mesh that led to the foreign body granuloma. after inguinal hernioplasty. In our case, the patient did not present with an infection and wedge biopsy revealed chronic inflammation [5]. Foreign body granuloma manifests on CT scan as a heterogenous mass which mimics a soft tissue tumour. The CT of our patient showed a fungating heterogeneous mass invading into the skin, subcutaneous and rectus abdominis muscle. In the case series reported by Hideaki et al, the CT scans did not reveal any mesh or suture that may be the cause of foreign body granuloma. Similarly, the CT scan of our patient did not reveal any mesh or suture.

Correlating to the suspicious findings on the CT scan, a wide local excision of the mass with clear margins was performed. Intra-operatively the mass was dissected meticulously leaving a rim of healthy surrounding tissue. On reaching the base of the mass we identified a piece of mesh that was firmly attached to the underlying tissues. The mesh was dissected away from healthy tissues (Figure 2A,B,C,D). A full histopathology report of the specimen confirmed the findings of a foreign body granuloma. Foreign body granuloma may occur in the absence of infection and presentation may mimic a soft tissue tumour. A tissue biopsy may guide us to prevent a radical excision which may lead to patient morbidity.

\section{Conclusion}

Foreign body granuloma may mimic a soft tissue tumour without the presence of post-operative infection.

\section{References}

1. Kise H, Shibahara T, Hayashi N, Arima K, Yanagawa M. Paravesical Granuloma after inguinal herniorrhaphy. Urol Int 1999; 62: 220-222

DOI: $10.1159 / 000030400$

2. Brown CN, Finch JG. Which mesh for hernia repair. Ann R Coll Surg Engl 2010; 92: 272-278

DOI:10.1308/003588410X12664192076296.

3. Nagar H. Stitch granulomas following inguinal herniotomy: a 10year review. J Paediatr Surg. 1993;28:1505-7.

DOI: http://dx.doi.org/10.1016/0022-3468(93)90442-N

4. Brandt WE: Unusual complications of hernia repairs. Am J Surg 1956;92:640-643.

DOI: http://dx.doi.org/10.1016/S0002-9610(56)80102-5

5. Birolini C, Minossi JG, Lima CF, Utiyama EM, Rasdan S. Mesh cancer: long -term mesh infection leading to squamous-cell carcinoma of the abdominal wall.

DOI: $10.1007 / \mathrm{s} 10029-013-1083-\mathrm{x}$

\section{Learning Points:}

- Occurrence of foreign body granuloma is rare with an incidence of $0.3 \%$ and 24 reported cases over past 60 years.

- Awareness should be made of the possible complication of inguinal hernia repair with mesh leading to foreign body granuloma

- Foreign body granuloma may present as a suspicious soft tissue tumour without any prior history of surgical site infection after inguinal hernia repair.

- Such cases should be treated as tumours whenever there is a doubt in diagnosis.

The Sri Lanka Journal of Surgery 2017; 35(3): 3-4 